\title{
A diagnostic algorithm for atypical spitzoid tumors: guidelines for immunohistochemical and molecular assessment
}

\author{
Jeong Hee Cho-Vega
}

Department of Pathology, Dermatopathology, University of Miami Miller School of Medicine, Miami, FL, USA

\begin{abstract}
Atypical spitzoid tumors are a morphologically diverse group of rare melanocytic lesions most frequently seen in children and young adults. As atypical spitzoid tumors bear striking resemblance to Spitz nevus and spitzoid melanomas clinically and histopathologically, it is crucial to determine its malignant potential and predict its clinical behavior. To date, many researchers have attempted to differentiate atypical spitzoid tumors from unequivocal melanomas based on morphological, immonohistochemical, and molecular diagnostic differences. A diagnostic algorithm is proposed here to assess the malignant potential of atypical spitzoid tumors by using a combination of immunohistochemical and cytogenetic/molecular tests. Together with classical morphological evaluation, this algorithm includes a set of immunohistochemistry assays (p16 ${ }^{\text {Ink4a }}$, a dual-color Ki67/MART-1, and HMB45), fluorescence in situ hybridization (FISH) with five probes (6p25, 8q24, 11q13, CEN9, and 9p21), and an array-based comparative genomic hybridization. This review discusses details of the algorithm, the rationale of each test used in the algorithm, and utility of this algorithm in routine dermatopathology practice. This algorithmic approach will provide a comprehensive diagnostic tool that complements conventional histological criteria and will significantly contribute to improve the diagnosis and prediction of the clinical behavior of atypical spitzoid tumors.
\end{abstract}

Modern Pathology (2016) 29, 656-670; doi:10.1038/modpathol.2016.70; published online 22 April 2016

The term atypical spitzoid tumor applies to a morphologically diverse group of rare melanocytic lesions. Majority of atypical spitzoid tumors occur in children and young adults, and less frequently in middle-aged or older adults. Atypical spitzoid tumor is characterized by a Spitz nevus morphology, but also displays histological features associated with melanoma. In fact, sometimes it can be nearly impossible to rule out spitzoid melanomas among these lesions and is one of the most challenging areas in Dermatopathology. Its exact biological potential is also controversial. However, the clinical implications of diagnosing malignancies are important, as failure to recognize melanomas can lead to inadequate excisions and recurrence of a neoplasm with the potential to metastasize. On the other hand, labeling a benign lesion as malignant can lead to morbidity from unwarranted wide re-excisions,

Correspondence: Dr JH Cho-Vega, MD, PhD, Department of Pathology, Dermatopathology The University of Miami Leonard M. Miller School of Medicine, 1611 N.W 12th Ave, Holtz Building ET Suite 2142A, Miami, FL 33136, USA.

E-mail: j.cho1@med.miami.edu

Received 8 November 2015; revised 28 February 2016; accepted 6 March 2016; published online 22 April 2016 sentinel lymph node biopsies, unnecessary adjuvant therapies, and emotional and financial strain to the family.

In 1948, it had become apparent that malignant melanomas in children have different clinical behavior than those in adults. ${ }^{1}$ This disparity in clinical behavior was obviously a matter of fundamental importance and Dr Sophie Spitz, a brilliant pathologist, raised the following questions: Do histologically malignant melanomas in children differ from those seen in adults? Can the clinical behavior of these lesions be predicted from their histologic findings? What factors, if any, influence the clinical behavior of these lesions? Should the melanomas of children be treated any differently from those of adults? She coined the term 'benign juvenile melanoma', which was later renamed as 'Spitz nevus' based on its benign clinical behavior.

Over the years, a subset of cases histologically similar to Spitz nevus cases demonstrated worrisome histological features associated with melanomas, displaying unexpected lymph nodal or distant metastasis during a long-term follow-up. These lesions, now called atypical spitzoid tumors, remain a controversial entity representing a histologically and clinically heterogeneous group of melanocytic 
Table 1 Histological and immunohistochemical features signifying melanoma in atypical spitzoid tumors

Architectural features
Asymmetry
Lack of circumscription $_{\text {Lack of maturation in depth of the dermis }}$
Ulcer $^{\mathrm{a}}$
High and frequent consumption of epidermis
Cytological features
Deep dermal mitosis ${ }^{\mathrm{a}}$
Frequent mitosis ${ }^{\mathrm{a}}$ and atypical mitosis in the dermis
High-grade cytological atypia

Host response
Brisk tumor infiltrating lymphocytic infiltrates
Immunohistochemical features
Deep dermal cell proliferation by a dual-color Ki67/MART-1
Complete loss of HMB45 expression
Complete loss of p16 expression

${ }^{\mathrm{a}}$ Histological features most correlated with disease progression. ${ }^{3}$

lesions with uncertain malignancy potential. There is no standardized histological criterion to establish the diagnosis of atypical spitzoid tumor, which results in occasional disagreement even among experts. ${ }^{2,3}$

To address these difficulties, appropriate molecular and immunohistochemical tests have been developed using a panel of selected immunomarkers and genes that contribute the most to melanoma pathogenesis. ${ }^{4-7}$ In particular, recent application of fluorescence in situ hybridization (FISH) and arraybased comparative genomic hybridization assays has uncovered an increasing spectrum of potential chromosomal aberrations implicated in atypical spitzoid tumors. Using a combination of immunohistochemical and cytogenetic/molecular tests, the diagnostic algorithm proposed here aims to assess the malignant potential of atypical spitzoid tumors.

\section{Proposed diagnostic algorithm}

In addition to classical morphological evaluation, this algorithm applies a set of immunohistochemistry assays (a dual-color of Ki67/MART-1, p16 ${ }^{\text {Ink4a }}$, and HMB45), FISH with five probes (covering the chromosomal loci 6p25, 8q24, 11q13, centromere 9, and 9p21; NeoSITE melanoma, Neogenomics Laboratories), and a more comprehensive arraybased comparative genomic hybridization assay (University of California, San Francisco) for an entire chromosomal evaluation.

Following the identification of histological features associated with melanoma including ulcers, asymmetry, high-grade cellular atypia, and frequent dermal and deep dermal mitosis from cases with Spitz nevus-like morphology (Table 1), a set of immunohistochemistry assays will be performed. If immunohistochemistry assays confirm deep dermal cell proliferation by a dual-color Ki67/MART-1, a complete loss of p16, and/or HMB45 (Table 1), an algorithmic FISH assay will be performed. Atypical spitzoid tumors positive by FISH for a 'melanoma pattern' of chromosomal aberrations, isolated homozygous loss of 9p21, isolated gain of $6 \mathrm{p} 25$, or gain of $11 \mathrm{q} 13$ will be diagnosed as 'spitzoid melanoma with a specific FISH signature' and further analysis by array-based comparative genomic hybridization will not be required (Figures 1a and c). For atypical spitzoid tumors with negative or borderline positive FISH results, the array-based comparative genomic hybridization assay will be performed. If the arraybased comparative genomic hybridization demonstrates a melanoma pattern of chromosomal aberrations, the cases will be diagnosed as spitzoid melanomas (Figure 1b). If the array-based comparative genomic hybridization results are negative or show a non-melanoma pattern of chromosomal aberrations, the case will be diagnosed as atypical spitzoid tumors (Figure 1d). As both FISH and arraybased comparative genomic hybridization assays do not detect known melanoma molecular signatures for this particular type of atypical spitzoid tumors, these cases can be associated with 'a low clinical risk,' as in cases of negative FISH. A conservative reexcision and long-term follow-up for cases with nonmelanoma pattern of array-based comparative genomic hybridization chromosomal aberrations is recommended.

\section{Histological evaluation}

Although Spitz nevus is a benign melanocytic neoplasm, its histological features are 'atypical' and can be difficult to distinguish from melanomas. HRAS activation found in a subset of Spitz nevi, either by amplification of chromosome $11 \mathrm{p}$ or by HRAS activation mutation, ${ }^{6}$ could give rise to incompletely transformed melanocytes that share several histological features with melanoma cells but have limited proliferative activity. Atypical spitzoid tumors display both Spitz nevus and spitzoid melanoma features. Therefore, it can be very difficult to rule out spitzoid melanoma in these lesions. In general, Spitz nevus morphology includes features such as dome-shaped, plaque-like, wedge-shaped morphology; little or no asymmetry; epidermal hyperplasia; junctional cleavage; presence of Kamino bodies; evidence of zonation/maturation deep in the dermis; and a population of enlarged epithelioid and/or spindled melanocytes with abundant opaque or glass cytoplasm. In contrast, morphological features signifying melanomas include asymmetry, lack of circumscription, lack of maturation deep in the dermis, ulcers, high and frequent consumption of epidermis, deep dermal mitosis, atypical and frequent mitosis in the dermal component, high-grade cytological atypia, and a brisk lymphocytic infiltrate (Table 1). There was low 


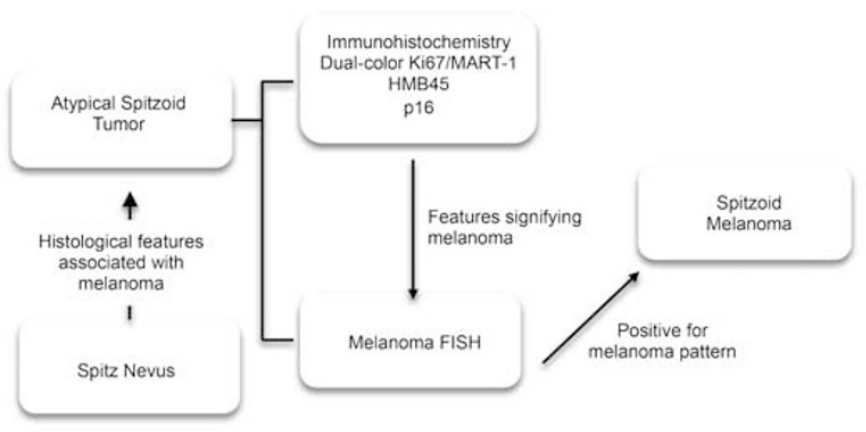

C

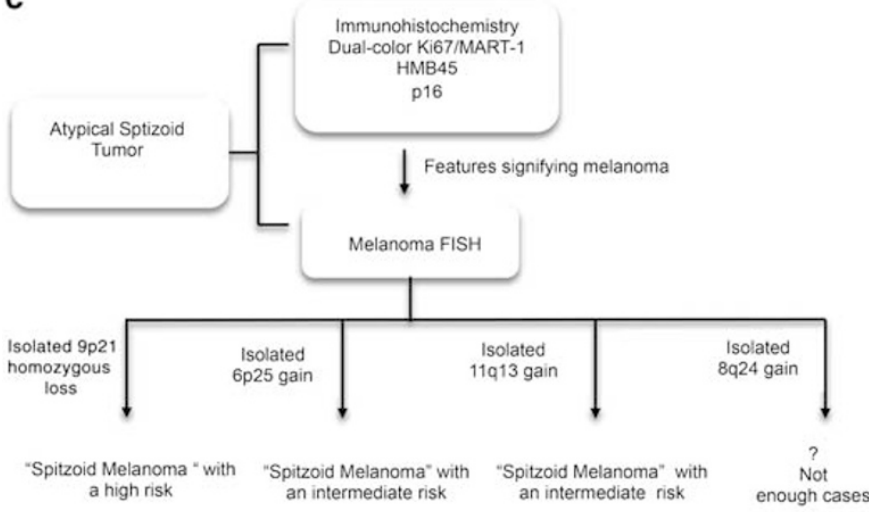

b

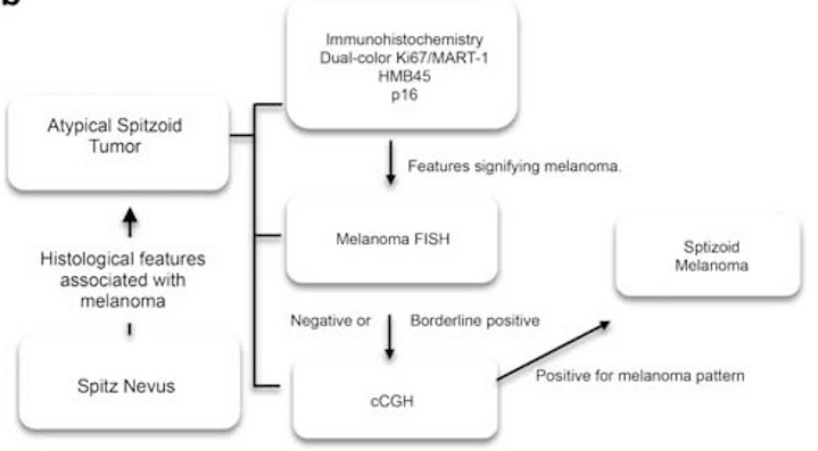

d

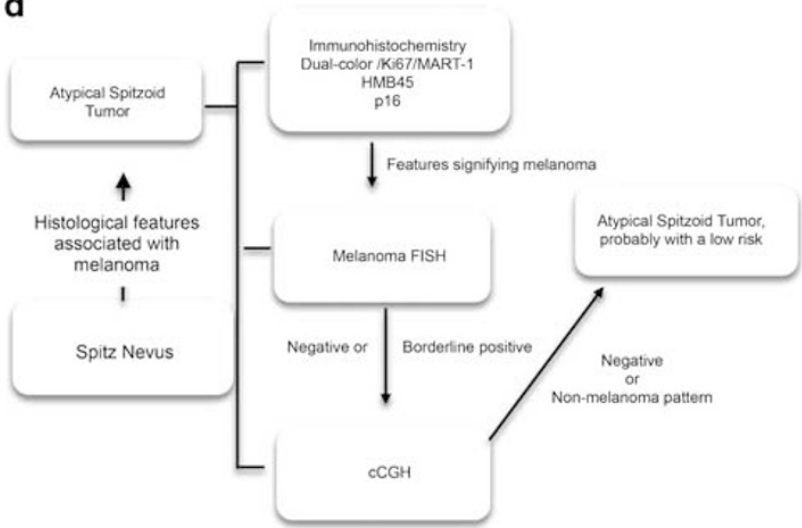

Figure 1 Four scenarios from the proposed diagnostic algorithm for the assessment of the malignant potential of atypical spitzoid tumors. In addition to classical morphological evaluation, this algorithm applies a set of immunohistochemical assays (a dual-color of Ki67/ MART-1 (Leica Biosystems), p16 Ink4a (Roche Life Sciences), and HMB45 (Leica Biosystems)), a fluorescence in situ hybridization (FISH) with five probes (6p25, 8q24, 11q13, centromere 9, and 9p21; NeoSITE melanoma, Neogenomics Laboratories), and an array-based comparative genomic hybridization (aCGH) assay (University of California, San Francisco).

inter-observer agreement among 13 experts grading the diagnostic morphological features for categorizing the lesions as malignant or nonmalignant spitzoid tumors in a large series of 75 cases. ${ }^{3}$ In this work, histological features most correlated with disease progression included frequent mitosis, deep mitosis, asymmetry, high-grade cytological atypia, and ulcer (Table 1). ${ }^{3}$

\section{Immunohistochemical tests}

Assessment of the expression of proteins involved in regulation of cell cycle and apoptosis regulators by immunohistochemistry (BAX, Ki67, Rb, p16, cyclin A, cyclin B1, p27, and p53) together with melanocytic markers (MART-1 and HMB45) has been a useful ancillary diagnostic tool to identify features of malignancy of melanocytic lesions. ${ }^{8-10}$ Although the interpretation of immunohistochemistry results remain subjective among pathologists and there is some sample selection bias, p16, Ki67, and HMB45 expression has shown relatively consistent differences between Spitz nevus and spitzoid melanoma (Table 2). ${ }^{9-14}$ Puri et $a 1^{14}$ demonstrated that the application of this immunohistochemistry panel significantly contributed to the diagnosis of spitzoid tumors and proposed that it could be a useful tool for classifying atypical spitzoid tumors.

\section{Cyclin-Dependent Kinase Inhibitor 2A (CDKN2A, p16 ${ }^{\text {Ink4a) }}$}

Using array-based comparative genomic hybridization, Curtin et $a l^{15}$ found that the most frequently lost genomic locus in melanomas was chromosome 9p. This gene locus was lost in $50 \%$ of conventional melanomas, and patients with familial melanoma syndrome are associated with a homozygous loss of the $C D K N 2 A$ gene. ${ }^{12,15}$

The p16 protein, encoded by the gene $C D K N 2 A$ on chromosome $9 p$, is critical to tumor suppression and has an important role in melanomas. ${ }^{16,17} \mathrm{p} 16^{\text {Ink4a }}$ is a cyclin-dependent kinase inhibitor that blocks formation of the catalytically active CDK4/6-cyclin D complex, thereby preventing the phosphorylation of the retinoblastoma $(R b)$ gene and passage through the cell cycle's G1/S checkpoint (Figure 2a). ${ }^{17-20}$ As a tumor suppressor, p16 is found in low levels in normal proliferating cells but events such as DNA damage, oncogenic stress, and aging trigger its 
Table 2 Comparison of the immunohistochemical expression of p16, Ki67, and HMB45 between Spitz nevi and spitzoid melanomas

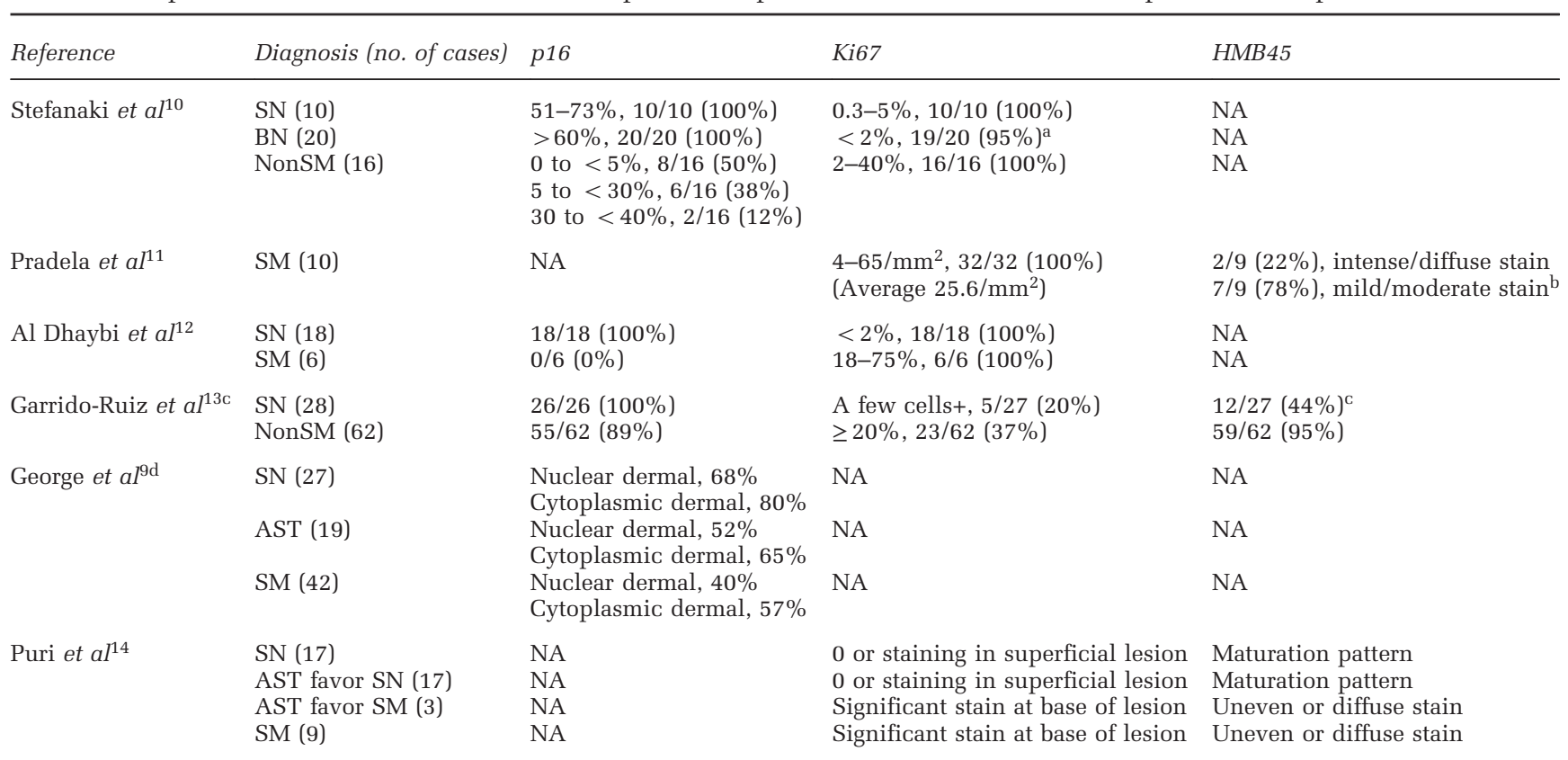

Abbreviations: BN, benign nevi; NA, not available; NonSM, nonspitzoid melanoma; SM, spitzoid melanoma; SN, Spitz nevus.

All references tested retrieval cases with consensus diagnosis.

${ }^{a}$ One case shows $5 \%$ Ki67 positivity.

b

CImmunohistochemistry was applied to tissue microarray.

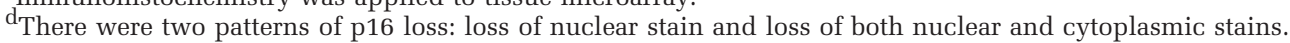

expression. ${ }^{17,20}$ The tumor suppressors $\mathrm{p} 14^{\mathrm{ARF}}$ and p16 $6^{\text {INK4A }}$ are coded from alternatively spliced transcripts in different reading frames at the INK4A/ARF locus on chromosome 9p21 (Figure 2a). The INK4A/ ARF locus on chromosome 9p21 is frequently altered in human cancer, and inherited $\mathrm{p} 16^{\text {Ink4a }} / \mathrm{p} 14^{\mathrm{ARF}}$ mutations are associated with melanoma susceptibility in $20-40 \%$ of familial melanomas. ${ }^{21}$

p16 protein expression can be detected by immunohistochemistry in formalin-fixed paraffin-embedded sections. Interestingly, the expression pattern of p16 protein detected by immunohistochemistry reflects its underlying molecular events within tumor cells (Figure 2b). Three patterns of p16 immunohistochemical protein expression were observed among spitzoid melanocytic tumors as follows: (1) homogeneous expression, (2) complete loss of expression, and (3) intratumoral heterogeneous loss of expression (Figure 2c). ${ }^{22}$ Homogeneous expression of p16 represents the underlying heterozygous loss of chromosome 9, 9p, or an intact chromosome 9 (Figure 2b). ${ }^{15}$ A complete loss of p16 expression represents either homozygous loss of chromosome 9 or $9 p$, a $p 16$ gene point mutation, or epigenetic events such as methylation of the $p 16$ gene promoter (Figure 2b). ${ }^{15}$ Intratumoral heterogeneous loss can be seen in tumors composed of mixed clones with either complete loss or homogeneous expression (Figure 2b). Several studies have shown similar observations; both dysplastic nevi and Spitz nevi display heterozygous loss of 9p21, whereas homozygous loss of 9p21 is common in spitzoid melanomas. ${ }^{23,24}$ Further, $67 \%$ of atypical spitzoid tumors with heterozygous loss of 9p21 showed p16 expression while none of the observed atypical spitzoid tumors with homozygous loss of 9p21 expressed p16 protein. ${ }^{25}$

This p16 immunohistochemical expression pattern can be applied to discriminate invasive melanomas from coexisting intradermal melanocytic nevi in melanoma excisions. In benign melanocytic nevi expressing the BRAF V600E mutation, overexpression of p16 has been observed and probably contributes to senescence and a state of arrested growth. ${ }^{20}$ Figure 2d illustrates one example of an intradermal nevus clearly demonstrating dense homogeneous expression of p16, whereas a homogeneous loss of p16 is observed in the invasive melanoma.

\section{Dual-Color Ki67/MART-1 Immunohistochemistry}

Melanoma progression is a multistep process initiated by uncontrolled proliferation of melanocytes. Melanocyte growth is associated with the actions of several proliferative proteins such as proliferating cell nuclear antigen, Ki67, minichromosome maintenance 2, and phosphorylated histone H3 (PHH3) and so on. 
a

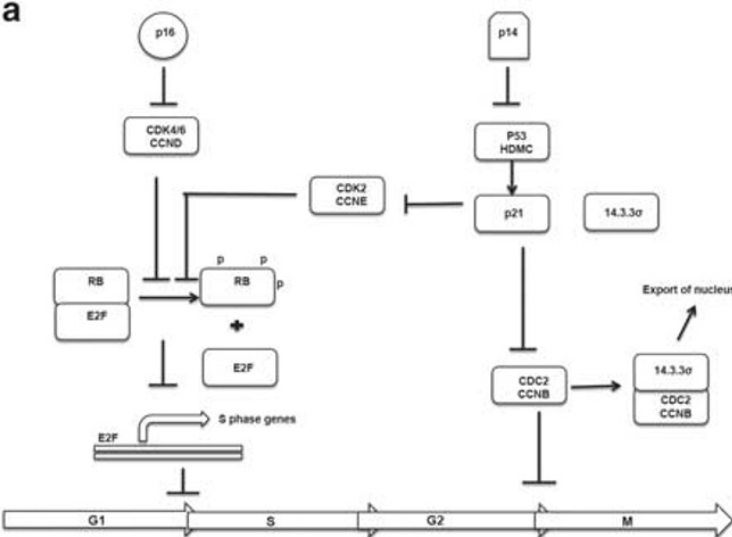

b

\begin{tabular}{ll}
\multicolumn{1}{c}{ Complete loss } & Homogeneous Expression \\
\hline Homozygous loss of Chr 9 or $9 p$ & Heterozygous loss of Chr 9 or $9 p$ \\
Methylation of promoter of $p 16$ gene & Intact chromosome 9 \\
Point mutation of $p 16$ gene & \\
\hline
\end{tabular}

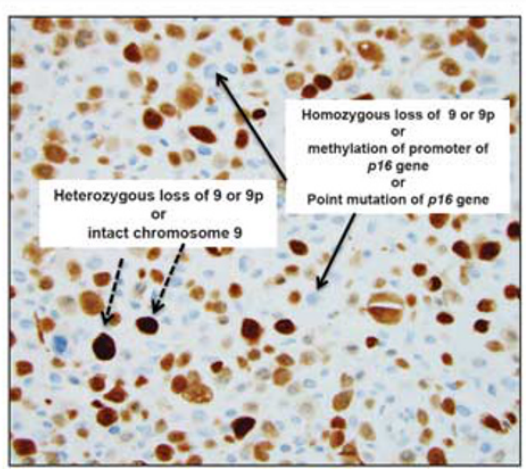

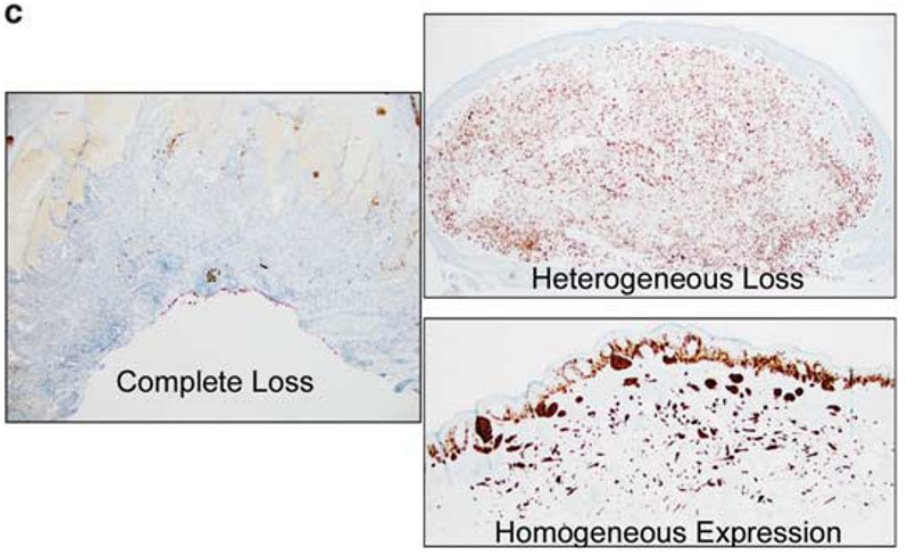

d
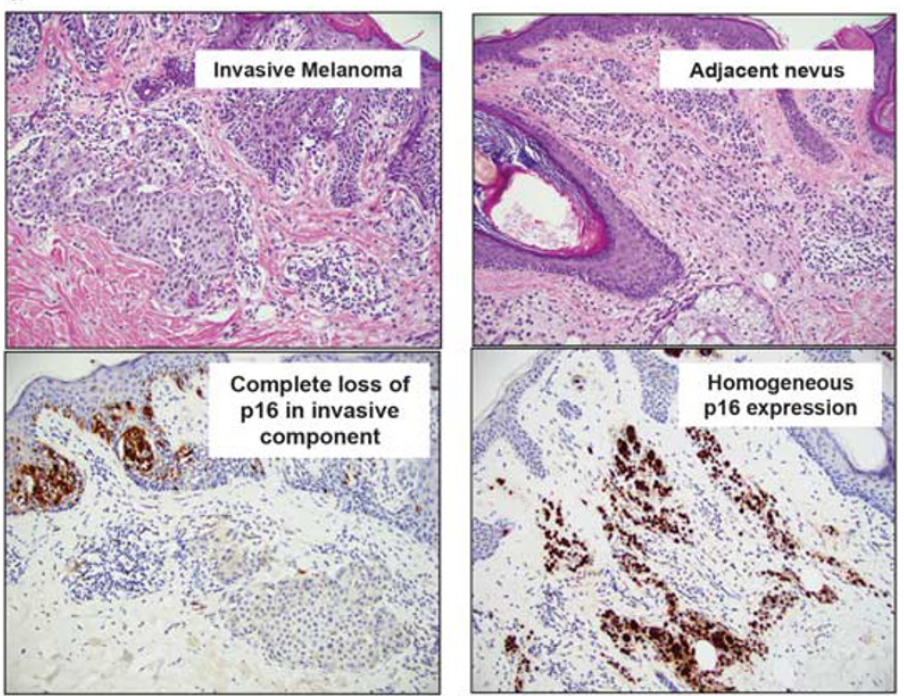

Figure 2 Immunohistochemical assessment of p16 ${ }^{\text {Ink4a }}$ expression. (a) Illustration of the regulatory roles of p16 ${ }^{\text {Ink4a }}$ and $p 14^{\text {ARF }}$ in the G1/ $\mathrm{S}$ and G2/M checkpoints of the cell cycle. (b) A summary of the various molecular mechanisms responsible for three characteristic immunohistochemical patterns of p16 protein expression. (c) Examples of three immunohistochemical patterns of p16 expression. A complete loss or an intratumoral heterogeneous loss was present in spitzoid melanomas. A homogeneous expression was found in a compound dysplastic nevus. (d) An example of immunohistochemical p16 expression patterns applied to distinguish between an invasive melanoma and an associated intradermal nevus.

Ki67, which is encoded by the MKI67 gene, is detected during all phases of the cell cycle $\left(\mathrm{G}_{1}, \mathrm{~S}, \mathrm{G}_{2}\right.$, and mitosis), except for the $\mathrm{G}_{0}$-phase (Figure 3a). It serves as a well established and widely used marker for cell proliferation and was proposed as a useful immunohistochemical marker for the distinction of Spitz nevus from spitzoid melanoma. ${ }^{8,26}$ In this algorithm, a dual-color Ki67 immunohistochemistry with a melanocytic marker specifically detects cell proliferation activity in melanocytes/melanoma cells by eliminating actively proliferating non-melanocytes such as reactive lymphocytes or endothelial cells or normal keratinocytes (Figure $3 \mathrm{~b}$ ). In this dual-color immunohistochemistry, melanocytes/melanoma cells with cell proliferation activity are highlighted by two colors (brown, nuclear Ki67 and red, cytoplasmic MART-1) and other actively proliferating non-melanocytes appear brown (nuclear Ki67 positivity; Figure 3b).
Ki67 positivity observed in the deep dermal melanocytes indicates increased cellular proliferation activity throughout the lesion, which correlates with a lack of maturation that is frequently observed in melanomas. ${ }^{8,22}$

This dual-color immunohistochemistry can be also applied for the differential diagnosis of capsular nevi from metastatic melanomas in the lymph node (Figure 3c). Usually the capsular nevi are highlighted by single MART-1 positivity reflecting lack of cellular proliferation activity and loss of HMB45 expression indicating maturation of melanocytes. Conversely, a dual positivity and HMB45 expression are detected at relatively high frequency in metastatic melanomas.

Histone $\mathrm{H} 3$ is a core histone protein that forms the major protein constituents of chromatin. Phosphorylation of the serine 10 residue of histone $\mathrm{H} 3$ is negligible during interphase but reaches a 

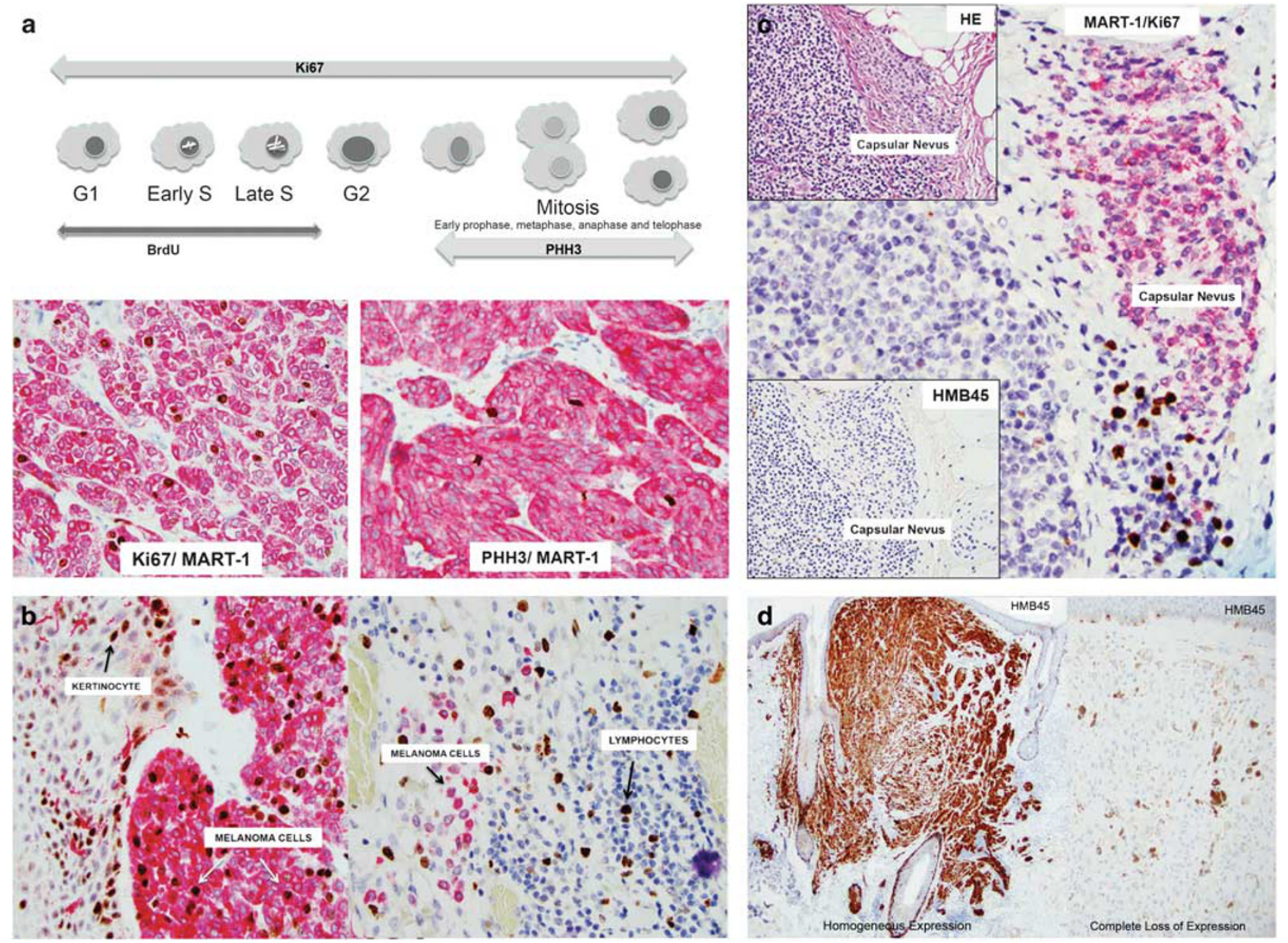

Figure 3 Immunohistochemical assessment of a dual-color Ki67/MART-1 and HMB45 expression. (a) Schematic representation of the expression of cell proliferation markers, Ki67, BrdU, and PHH3, during the cell cycle (top). 5-Bromo-2'-deoxyuridine (BrdU) is incorporated into the DNA only during the S-phase of the mitotic process and identifies cells that have replicated DNA. A dual-color Ki67/MART-1 and a dual-color PHH3/MART-1 immunohistochemistry in a melanoma (bottom). (b) Melanocyte-specific cell proliferation activity detected by dual-color Ki67/MART-1 immunohistochemistry. (c) A dual-color Ki67/MART-1 and HMB45 immunohistochemistry applied for the differential diagnosis of capsular melanocytic nevus from metastatic melanoma in the lymph node. (d) Two distinct immunohistochemical HMB45 expressions in metastatic melanomas; a homogeneous expression or a complete loss of expression.

maximum for chromatin condensation during mitosis (Figure 3a). PHH3 is an antibody that specifically detects the phosphorylated $\mathrm{H} 3$ (at serine 10 or serine 28) core histone protein. PHH3 is typically not detectable once mitosis is completed (Figure 3a) and it serves as a reliable marker for identifying mitotic activity, as it is not expressed in apoptotic bodies-common morphologic mimics of mitotic figures in routine histological sections. ${ }^{27}$ The application of a dual-color PHH3/MART-1 immunohistochemistry increases the sensitivity and accuracy of detecting melanocyte-specific mitotic figures (Figure 3a); it reduces inter-observer variability and the time required to identify mitoses in invasive melanomas. ${ }^{28}$ For the diagnostic algorithm proposed here, a dual-color PHH3/MART-1 immunohistochemistry can be used for atypical spitzoid tumors with dermal components when routine histological sections fail to demonstrate dermal mitosis.

\section{Human Melanoma Black-45}

Human Melanoma Black45 (HMB45) antibody was derived from an extract of a pigmented melanoma involving the lymph node. ${ }^{29}$ Two distinct HMB45 immunohistochemical patterns can be observed in melanomas, including a homogeneous expression in metastatic melanomas and a complete loss of expression in both metastatic and invasive melanomas (Figure 3d). In benign nevi, HMB45 expression yields a stratified pattern (maturation pattern) with fewer positive cells or a negative reaction in the depth of the dermis. ${ }^{30}$

In summary, in conjunction with atypical histological features of atypical spitzoid tumors, immunohistochemical detection of deep dermal cell proliferation using a dual-color Ki67/MART-1 stain and a complete loss of p16 and/or HMB45 expressions are the signifying features of melanoma (Table 1). However, it is important to understand 

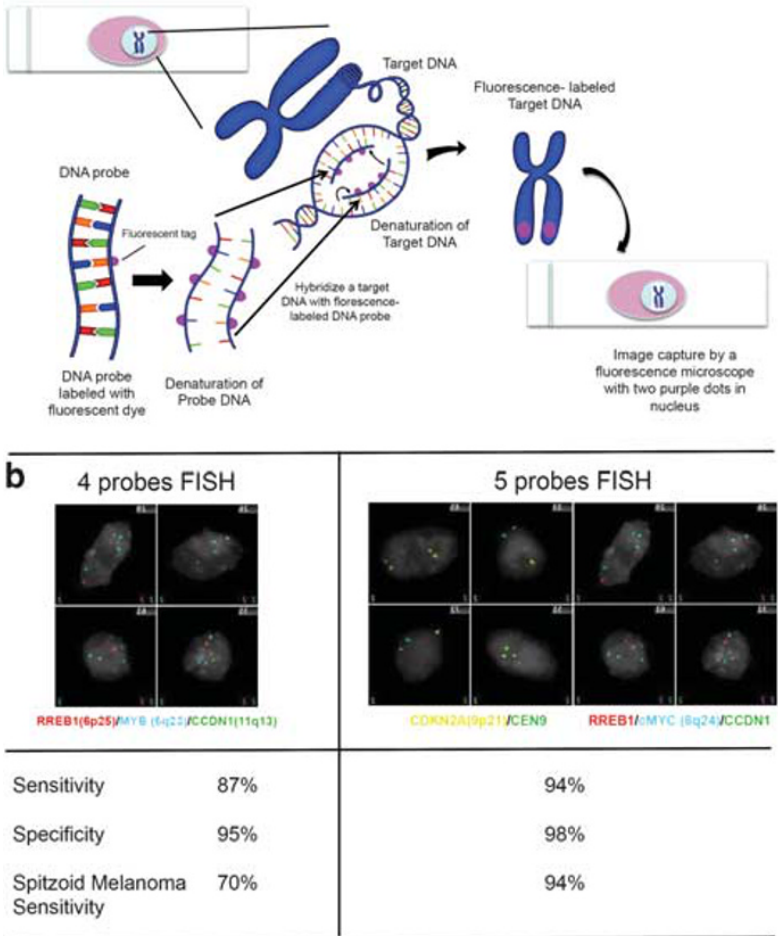

C

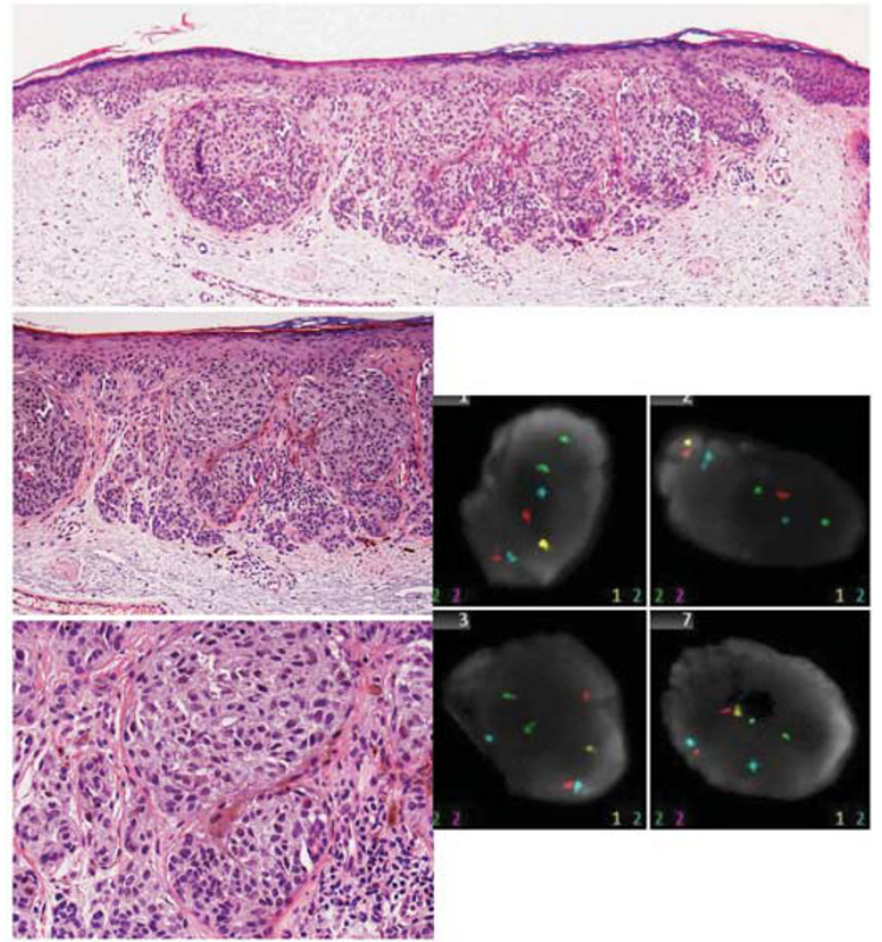

Figure 4 Cytogenetic assessment using fluorescence in situ hybridization (FISH). (a) Schematic representation of FISH technique. (b) Comparison between the initial 4-probe and the current 5-probe FISH assays. The cutoff for positive 5-probe FISH results: $>29 \%$ cutoff for all 5 probes. The cutoff for negative FISH results: 6p25<16\%, 8q24<10\%, p16/CEN9 < 10\%, and 11q13<19\%. (c) An example of ambiguous nonspitzoid compound melanocytic tumor with an isolated loss of $6 q 23$ in a 58-year-old man's back. The 4-probe FISH assay revealed an isolated loss of 6q23 and the case was diagnosed as a 'melanoma' in 2010.

that the complete loss of p16 or HMB45 expression alone is a crude indicator for the diagnosis of melanoma. Thus, it is essential to perform further cytogenetic/molecular tests for p16 and correlate these immunohistochemical results with the morphological findings.

\section{Diagnostic cytogenetic and molecular tests}

Recent advances in the molecular pathogenesis of melanocytic proliferation have revealed many genetic differences between benign nevi and melanomas. Most benign nevi are driven by point mutations in selected oncogenes, but they do show gross chromosomal abnormalities in exceptional cases. ${ }^{5}$ In contrast, tumor progression from a nevus to a melanoma is associated with chromosomal instability resulting in gains, amplifications, and/or loss of specific chromosomal material that can be detected by genetic techniques. These molecular advances can be exploited to develop powerful objective diagnostic molecular tests as promising tools to complement the histological assessment of ambiguous melanocytic tumors. Among these methods, FISH and array-based comparative genomic hybridization assays have become increasingly powerful supplements to provide useful diagnostic and prognostic information in borderline melanocytic lesions, including atypical spitzoid tumors. Additionally, these techniques are being used to identify an increasing spectrum of potential chromosomal aberrations in atypical spitzoid tumors.

\section{Melanoma FISH}

FISH is used in the diagnosis of melanoma to detect a selected number of chromosomal copy number aberrations and is emerging as a powerful tool to assess the malignant potential of atypical spitzoid tumors.

Principle of FISH technique. FISH assay begins with hybridization of FISH probes (short DNA fragments) to a $5 \mu \mathrm{m}$-thick formalin-fixed paraffinembedded section of a tumor of interest (Figure 4a). Under a fluorescence microscope, each fluorescently labeled DNA probe that hybridizes to a tumor cell nucleus appears as a distinctly colored fluorescent dot (Figure 4a). Each dot identifies a single copy of the chromosomal locus and each diploid nucleus will show two dots of the same color. If a DNA region of interest is gained or deleted, more than two dots or less than two dots will be observed, respectively. The number of specific florescent colored dots per 
Table 3A Chronological comparison of the sensitivity and specificity of 4- and 5-probe FISH assays for the diagnosis of melanoma

\begin{tabular}{|c|c|c|c|c|c|}
\hline \multirow{2}{*}{ Reference } & \multirow{2}{*}{ No of cases ${ }^{\mathrm{a}}$} & \multicolumn{2}{|c|}{4 FISH probes } & \multicolumn{2}{|c|}{5 FISH probes } \\
\hline & & Sensitivity (\%) & Specificity (\%) & Sensitivity (\%) & Specificity (\%) \\
\hline Gerami et $a l^{31}$ & 497 & 87 & 95 & & \\
\hline Gerami et al ${ }^{38}$ & 233 & 83 & 94 & & \\
\hline Vergier et $a l^{36}$ & 43 & 85 & 90 & & \\
\hline $\begin{array}{c}\text { Kerl et } a l^{37} \\
\text { Abbott } \\
\text { Gerami }\end{array}$ & 163 & $\begin{array}{l}82 \\
83\end{array}$ & $\begin{array}{l}94 \\
92\end{array}$ & & \\
\hline Gammon et al ${ }^{34}$ & $43^{\mathrm{b}}$ & 70 & & 85 & \\
\hline Gerami et $a l^{32}$ & 424 & & & 94 & 98 \\
\hline
\end{tabular}

4-Probe FISH assay includes RREB1, CEP6, MYB, and CCND1. For the cutoff for positive FISH results, at least one of the following four criteria must be fulfilled. Abbott (Abbott, Wiesbaden, Germany) criteria: (1) $\geq 2.5$ average of CCND1 (cyclin D1) signal per nucleus; (2) $\geq 2.5$ average of $M Y B$ signal per nucleus; ( 3 ) $\geq 31 \%$, loss of $M Y B$ relative to CEP6; or (4) $\geq 63 \%$, $\leq$ or $\geq 2$ signal per nucleus for $R R E B 1$. Gerami criteria: (1) $>38 \%$, $>2$ CCND1 signal per nucleus; (2) > 40\%, the number of $M Y B$ signal minus the number of the CEP6 signal; (3) > 55\%, the number of RREB1 signal plus the number of the CEP6 signal; or (4) > 29\%, > 2 RREB1 signals per nucleus. 5-Probe FISH assay includes RREB1, $c M Y C$, $C D K N 2 A$, CEN9, and $C C N D 1$. The cutoff for positive FISH results was $>29 \%$ cutoff for all probes. The lower cutoff for negative FISH results: $R R E B 1$ (6p25) = 16\%, $C M Y C$ $(8 \mathrm{q} 24)=10 \%, C D K N 2 A(\mathrm{p} 16)(9 \mathrm{p} 21) / \mathrm{CEN} 9=10 \%$, or $C C N D 1(11 \mathrm{q} 13)=19 \%$.

${ }^{\mathrm{a}}$ Cases include mixed various benign nevi and melanomas.

${ }^{\mathrm{b}}$ All 43 cases were spitzoid melanomas.

nucleus can then be counted either manually or with software designed for automated analysis. Due to the intrinsic variability of FISH signals, this technique requires the examination of sufficient number of tumor cells and strict quality control measures.

Initial 4-probe FISH assay for diagnosis of melanoma. The initial set of FISH probes for melanoma was derived from a combinatorial analysis of the array-based comparative genomic hybridization data set of Bastian et $a l^{4-6}$ showing the best discriminatory difference between melanomas and nevi. Fourteen FISH probes were assembled from 13 regions on eight different chromosomes $(1,6,7,9,10,11,17$, and 20) from the array-based comparative genomic hybridization database and one additional probe targeting KIT on chromosome 4 as a potential therapeutic target. FISH probes targeting oncogenes were selected from the region of commonly gained chromosomal loci and tumor suppressor gene targets were selected from the regions of commonly deleted chromosomal loci.

In 2009, validation of 97 melanomas and 95 nevi yielded a combination of 4 initial FISH probes (ras responsive element binding protein 1 (RREB1, 6p25), v-myb myeloblastosis viral oncogene homolog (MYB, 6q23), centromere 6 (CEN6), and cyclin D1 (CCND1, 11q13)). This combination was identified as having the best-combined discriminatory ability for distinguishing melanomas from benign nevi (Figure 4b). ${ }^{31}$ Analyzing a second cohort of 58 melanomas and 51 nevi and a third cohort of 83 melanomas and 86 nevi, this probe set demonstrated discriminatory power with a sensitivity of $86.7 \%$ and specificity of $95.4 \%$ (Figure 4b). ${ }^{32}$ The application of this 4-probe FISH assay, performed as part of routine clinical tests, demonstrates a significant reduction in the number of equivocal diagnoses for various ambiguous melanocytic neoplasms, particularly if FISH results are positive. ${ }^{33}$

However, the sensitivity of this probe set was relatively low, $\sim 70 \%$, for detecting spitzoid melanomas (Figure 4b). As the most commonly lost genomic region in melanomas and spitzoid melanomas was chromosome $9 p$ from the array-based comparative genomic hybridization database, ${ }^{15}$ it was unclear as to why chromosome $9 p$ probe had not been selected in this set of 4 probes. It turns out that heterozygous losses of 9p21 were enumerated in both melanomas and nevi in the early stage of probe selection data, and as a result, the 9p21 probe failed to demonstrate a statistically significant difference between melanoma and nevi. ${ }^{34}$

Current 5-probe FISH assay for diagnosis of melanoma. In 2012, after testing three training sets using 322 tumors, including 152 melanomas and 170 nevi, the initial FISH panel has been redefined to the currently used 5-probe set of 6p25, 9p21, CEN9, and 11q13, and myelocytomatosis oncogene (MYC, 8q24) (Figure 4b). ${ }^{34}$ This probe set was validated on 51 melanomas and 51 nevi, and displayed improved discriminatory power with a sensitivity of $94 \%$ and specificity of $98 \%$ and significantly improved sensitivity of spitzoid melanoma detection to $94 \%$ (Table $3 \mathrm{~A}$ and Figure $4 \mathrm{~b}$ ). ${ }^{32}$

Table 3A summarizes the chronological improvement in sensitivity and specificity of the 4- and 5-probe FISH assay for the diagnosis of melanomas and demonstrates relatively consistent results between American and European groups even when different cutoff criteria were applied. ${ }^{31,32,34-38}$ 
FISH-based risk assessment for atypical spitzoid tumors. For atypical spitzoid tumors, the FISH assay is not only diagnostic but can also provide clinically useful risk assessment data. Massi et al ${ }^{39}$ previously documented the utility of 4-probe FISH for the prognostic evaluation of atypical spitzoid tumors. In their study, among the six FISH-positive cases, two patients showed disease progression, one with visceral metastasis and death, and another with in-transit metastasis. In contrast, none of the 19 cases with a negative FISH had disease progression or metastasis. ${ }^{39}$

The FISH-based risk assessment for atypical spitzoid tumors applied to this algorithm was derived from a retrospective data set of Gerami et $a l^{40}$ showing a striking difference in clinical risk according to the subtype of the isolated chromosomal aberrations in atypical spitzoid tumors. However, this data set is very limited and additional studies with a larger number of cases and longerterm follow-up are warranted to further substantiate its significance.

Followings are the characteristics of those distinct subtypes of spitzoid melanomas and atypical spitzoid tumors with specific isolated chromosomal aberrations and its associated clinical risks as observed in this work.

1. Atypical spitzoid tumors with an isolated loss of $6 q 23$ were frequently associated with a positive sentinel lymph node (6 of 11 cases, 55\%) but demonstrated infrequent nonsentinal node metastasis, palpable adenopathy, and in-transit or distant metastasis. ${ }^{41}$ Histologically, the tumors were characterized by minimal pagetoid spread, expansile nodular growth, and focal ulceration. Therefore, it was suggested that a diagnosis of melanoma should not be given for this subset of atypical spitzoid tumor and at least provisionally referring to them as 'atypical spitzoid tumors with isolated loss of 6q23' that probably has a clinically low risk similar to negative FISH atypical spitzoid tumors. As a result, the $6 q 23$ probe was replaced with $8 q 24$ in the current 5-probe FISH assay.

The above atypical spitzoid tumor data also led us to reconsider the diagnostic significance of an isolated loss of 6q23 for nonspitzoid borderline melanocytic tumors in adults. In 2010, we observed a case with an atypical compound melanocytic tumor with architectural disorder in the back of 58 years old man (Figure 4c). An unusually confluent and large junctional proliferation of melanocytes, subtle asymmetry, dermal mitosis, and poor circumscription were observed, which were histologically worrisome features. Conversely, a low-grade cytological atypia, lack of pagetoid spreading, mature in depth of the dermis, and non-brisk host response were the benign features (Figure 4c). The 4-probe melanoma FISH available at that time revealed an isolated loss of 6q23 and the lesion was diagnosed as a 'melanoma' (Figure 4c). The patient was treated with a melanoma protocol. As it has now become clear that the isolated loss of 6q23 is one of the lesser aggressive molecular signatures in atypical spitzoid tumors, this patient might have been over-diagnosed and treated too aggressively. This presented case may also provide evidence that an isolated loss of $6 q 23$ is evolving as a molecular signature, even for the detection of nonspitzoid borderline melanocytic tumors of adults.

2. Atypical spitzoid tumors carrying an isolated homozygous loss of 9p21 metastasized beyond the sentinel lymph node (6/11 cases, 55\%) and caused death due to disease (3/11 cases, 27\%). ${ }^{40}$ A homozygous loss of 9p21 was detected in majority of tumor cells in these cases (33-100\%, average $80 \%$ ). In contrast, a homozygous loss of 9p21 was found only in 3 of 64 control cases (5\%) and progressed with a benign clinical course for 5 years. Hence, a diagnosis of 'spitzoid melanoma with isolated homozygous loss of 9p21' was proposed and characterized to be less fatal than spitzoid melanomas with a classic melanoma pattern of chromosomal aberrations but a high clinical risk and has a higher incidence in children than in adults (Figure 1c). ${ }^{25,40}$ These tumors also displayed histological characteristics of severe cytological atypia, dominantly epithelioid cytomorphology, frequent dermal mitosis, a complete loss of p16 protein expression on immunohistochemistry, and the presence of BRAF mutation in a small subset of tumors. ${ }^{25}$

3. Atypical spitzoid tumors carrying isolated heterozygous loss of 9p21 were characterized by more benign features, including absence of distant metastasis, less cytological atypia, less epithelioid cytology, less dermal mitosis, the presence of p16 protein expression by immunohistochemistry in $67 \%$ of patients, and BRAF mutation in none of these tumors and showed a good prognosis similar to that of atypical spitzoid tumors with an isolated loss of 6 q23 or negative FISH. ${ }^{25,34,40}$

4. Atypical spitzoid tumors with an isolated gain of 6 p25 or gain of $11 q 13$ were provisionally designated as diagnosis of 'spitzoid melanoma with an isolated gain of 6p25 or gain of 11q13' and demonstrated a higher risk for aggressive behavior compared with atypical spitzoid tumors that are FISH negative, but lesser than those that display homozygous loss of 9p21 (Figure 1c). ${ }^{40}$

5. Atypical spitzoid tumors with an isolated gain of 8q24 are too rare to evaluate its risk although an infrequent copy number aberration in spitzoid neoplasms has been reported. ${ }^{40}$

In summary, this reported FISH-based risk assessment data revealed that (1) a higher risk in spitzoid melanomas with an isolated homozygous loss of 9p21 but less fatal than spitzoid melanomas with classic melanoma pattern of chromosomal aberrations, (2) an intermediate risk in spitzoid melanomas with an isolated gain of 6p25 or gain of 11 q13, and (3) a low risk in atypical spitzoid tumors with an isolated heterozygous loss of 9p21 or an isolated loss of 6 q23 or negative FISH. In this algorithm, these 


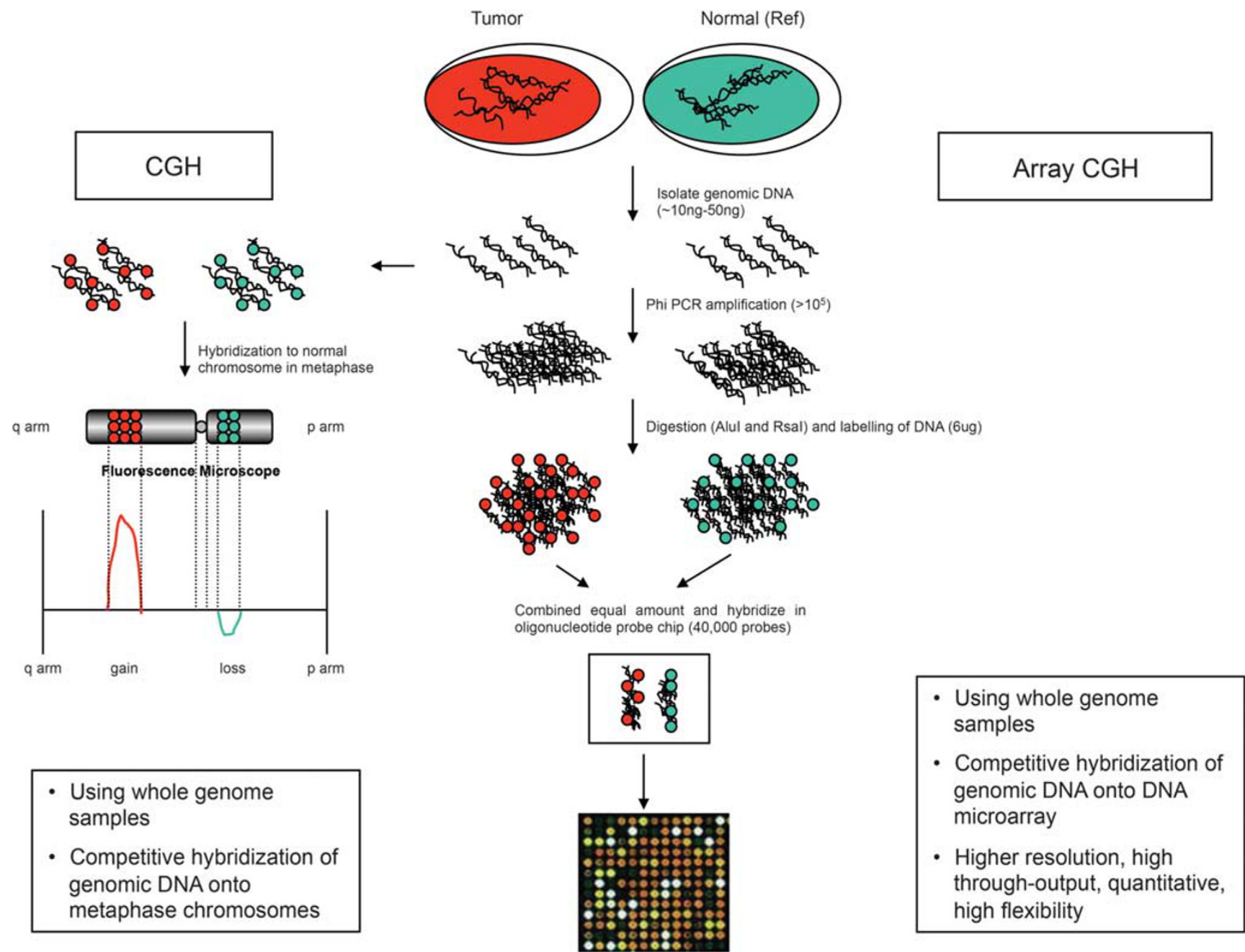

Figure 5 Schematic representation of the comparison between conventional comparative genomic hybridization (CGH; left) and arraybased CGH (aCGH; right).

high risk-related FISH signatures are reported and for those cases, array-based comparative genomic hybridization is not applied further (Figure 1c).

\section{Array-Based Comparative Genomic Hybridization}

In 1992, a novel technique, comparative genomic hybridization (CGH), was introduced to detect chromosomal copy number aberrations throughout the genome of tumor cells in a single experiment (Figure 5).42 A chip-based comparative genomic hybridization approach with high resolution and accuracy, known as array-based CGH (aCGH; Agilient Technologies), was subsequently developed in 2004 (Figure 5). ${ }^{42}$ This assay includes 40000 probes that span the human genome. In comparison with a conventional comparative genomic hybridization, ${ }^{43}$ aCGH has several advantages, such as: this method can identify regions of genomic imbalance more accurately, it has a much higher resolution, allowing the identification of tumor-related genes, and can be applied to formalin-fixed paraffin-embedded tissues. For these reasons, array-based comparative genomic hybridization has contributed to the discovery of genetic changes characteristic of spitzoid melanocytic neoplasms. ${ }^{44}$

Over the next decade, Bastian et al showed that melanomas have a variety of chromosomal copy number aberrations including copy number gains of $1 \mathrm{q}, 6 \mathrm{p}, 7,8 \mathrm{q}, 17 \mathrm{q}$, and $20 \mathrm{q}$ and losses of chromosomes 6q, 8p, 9p, and 10q. ${ }^{4,53}$ Among the 54 nevi evaluated, only 7 lesions (13\%), all of them Spitz nevi, had the same isolated gain in the entire short arm of chromosome 11, near the location of the HRAS gene, or an isolated loss of a portion or whole of chromosome 3 involving the region (3p21) where the $B A P 1$ gene is located (Table $3 \mathrm{~B})^{4,5}$ This lack of significant overlap in the patterns of chromosomal aberrations in melanomas and nevi has provided a rationale for using aCGH as an ancillary diagnostic tool for detecting malignancies for ambiguous melanocytic tumors including atypical spitzoid tumors (Table 3B). ${ }^{4,5,43,45-47}$ 
Table 3B Comparison of the frequency of chromosomal aberrations detected by CGH or aCGH in Spitz nevi, benign nevi, and melanomas

\begin{tabular}{|c|c|c|}
\hline Reference & Diagnosis (no. of cases) & $\%$ of chr aberrations \\
\hline Bastian et $a l^{43}$ & NonSM (32) & $94 \%(30 / 32)$ \\
\hline Bastian et $a l^{4}$ & SN (17) & $\begin{array}{l}0 \%(13 / 17) \\
18 \%(3 / 17), \text { gain of } 11 p \\
6 \%(1 / 17), \text { gain of } 7 q 21\end{array}$ \\
\hline Bastian et $a l^{5}$ & $\begin{array}{l}\text { BN (47) } \\
\text { SN (7) } \\
\text { NonSM (132) }\end{array}$ & $\begin{array}{l}0 \%(0 / 47) \\
100 \%(7 / 7), \text { all gain } 11 p \text { (as single aberration) } \\
96 \%(127 / 132), \text { multiple aberrations }{ }^{\mathrm{a}}\end{array}$ \\
\hline Ali et $a l^{45}$ & $\begin{array}{l}\text { SN (8) } \\
\text { Spitz tumor (1) } \\
\text { SM (1) }\end{array}$ & $\begin{array}{l}13 \%(1 / 8), \text { gain of both } 7 q \text { and } 11 p \\
100 \%(1 / 1) \text {, gain of } 19 p \\
100 \%(1 / 1) \text { gain of } 6 p \text { and } 17 q \text { : loss of } 1 p \text { and } 15 p\end{array}$ \\
\hline Raskin et $a l^{47}$ & $\begin{array}{l}\text { SN (8) } \\
\text { AST (16) }\end{array}$ & $\begin{array}{l}13 \%(1 / 8), \text { gain of } 11 \mathrm{p} \\
0 \%(9 / 16) \\
44 \%(7 / 16), \text { multiple aberrations }\end{array}$ \\
\hline Wang et al ${ }^{46}$ & $\begin{array}{l}\text { SM (2) } \\
\text { BN (5) } \\
\text { NonSM (25) }\end{array}$ & $\begin{array}{l}100 \%(2 / 2), \text { loss of } 9 \text { and } 11 \text {; gain of } 8,9 \text {, and } 11 \\
0 \%(0 / 5) \\
92 \%(23 / 25), \text { multiple aberrations }{ }^{\mathrm{a}}\end{array}$ \\
\hline
\end{tabular}

Abbreviations: AST, atypical spitzoid tumor; BN, benign nevus; NonSM, nonspitzoid melanoma; SN, Spitz nevus; SM, spitzoid melanoma. Studies 43, 4, and 5 used CGH; 45, 46 and 47 used aCGH.

a The most frequent gains were of chr 6p, 1q, 7p, 7q, 8q, 17q, and 20q. The most frequent losses were of chr 9p, 9q, 10q, 10p, 6q, and 11q.

${ }^{b}$ Gains of chr 1p, 3, 8, 9, and 20p; losses of chr 1, 3, 4p, 5q, 7q, 8, 9, 14q, and 18q.

For the array-based comparative genomic hybridization technique, 5-10 shaves of $25 \mathrm{~mm}$ thick paraffin sections per sample are required for one assay and the tumor cells are microdissected from the specimen to collect a relatively pure tumor population, if necessary. The target DNA then needs to be isolated and purified from the tumor sample, labeled with a fluorochrome, and hybridized (Figure 5). The copy number change must be observed in at least $30-50 \%$ of the collected cells and if an aberration is identified, the results are typically very specific. aCGH rarely yields false negativity, usually when the samples examined contain $<20-30 \%$ of tumor cells. ${ }^{5}$

For the diagnostic algorithm proposed here, one essential question was that which cytogenetic/molecular test, either FISH or array-based comparative genomic hybridization, should be performed first for individual cases of atypical spitzoid tumors. A FISH assay that detects selected chromosomal aberrations, is preferred as the first molecular test in this algorithm, as it is simple, rapid, relatively inexpensive, applied to standard $5 \mu \mathrm{m}$-thick paraffinembedded sections, identifies even small clones of chromosomally aberrant cells within a larger tumor, and allows histopathologic correlation.

However, FISH is not as comprehensive as aCGH. A maximum of four chromosomal targets can be evaluated by a single FISH whereas aCGH can be used to analyze whole chromosomal copy number aberrations in a single experiment. This often generates discrepancy between FISH and aCGH results (Figures 6 and 7). ${ }^{35}$ The main drawbacks of aCGH are that it is an expensive, laborious, and timeconsuming procedure.

In summary, both FISH and aCGH assays applied to the current diagnostic algorithm for atypical spitzoid tumors are highly sensitive and specific for melanomas. Ninety percent of overall concordance was found using both FISH and aCGH assays, consistent with a recent report indicating that both assays should be used as complementary methods to improve the accuracy of genetic evaluation of melanocytic tumors. ${ }^{46}$ However, it is critical to understand potential pitfalls of these assays and consider cytogenetic/molecular results as a diagnostic adjunct to routine histological and immunophenotypical findings, and not as a stand-alone diagnostic test.

Several kinase fusions of ROS, NTRK1, ALK, BRAF, and RET were recently discovered as additional molecular alterations found in the entire biological spectrum of spitzoid tumors, including $55 \%$ of Spitz nevi, $56 \%$ of atypical spitzoid tumors, and $39 \%$ of spitzoid melanomas. ${ }^{48}$ A subset of somatic atypical spitzoid tumors (28\%) showed loss of BAP1 expression and concomitant BRAF mutations. ${ }^{49}$ These molecular alterations are not diagnostic for a melanoma and FISH assays are not applied for the detection of kinase gene fusions in the current diagnostic algorithm for atypical spitzoid tumors.

Recently, two additional molecular tests using reverse transcription PCR (RT-PCR) were developed. The 'Myriad myPath melanoma' (Myriad Genetics) is a diagnostic test for borderline melanocytic lesions using quantitative reverse transcription polymerase chain reaction. It is based on the expression profile of 23 genes including preferentially expressed antigen in melanoma (PRAME), a group of S100 genes (A7, A8, A9, and A12) and P13, a group of eight genes involving tumor immune response signaling, and nine housekeeping genes. ${ }^{50}$ The 'DecisionDx-Melanoma' test (Castle Bioscience) is designed to predict 5 -year metastasis risk by measuring expression levels of 31 genes. This test stratifies patients as Class 1 


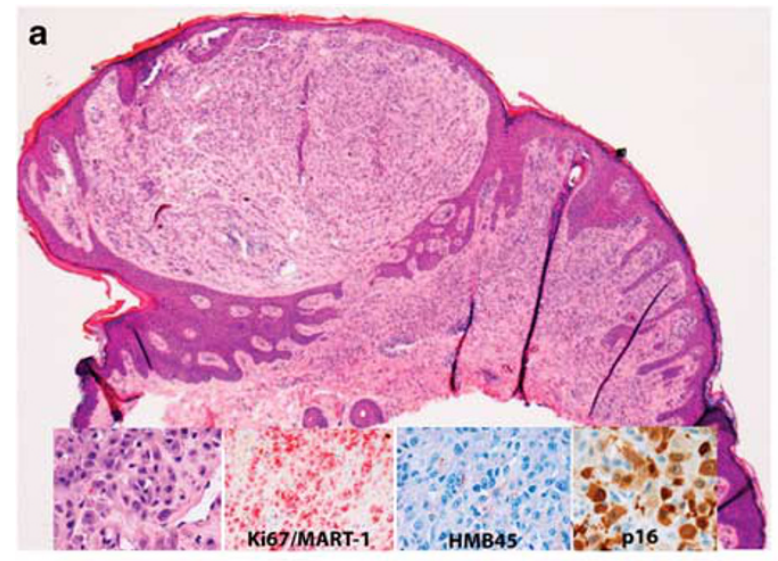

C

b
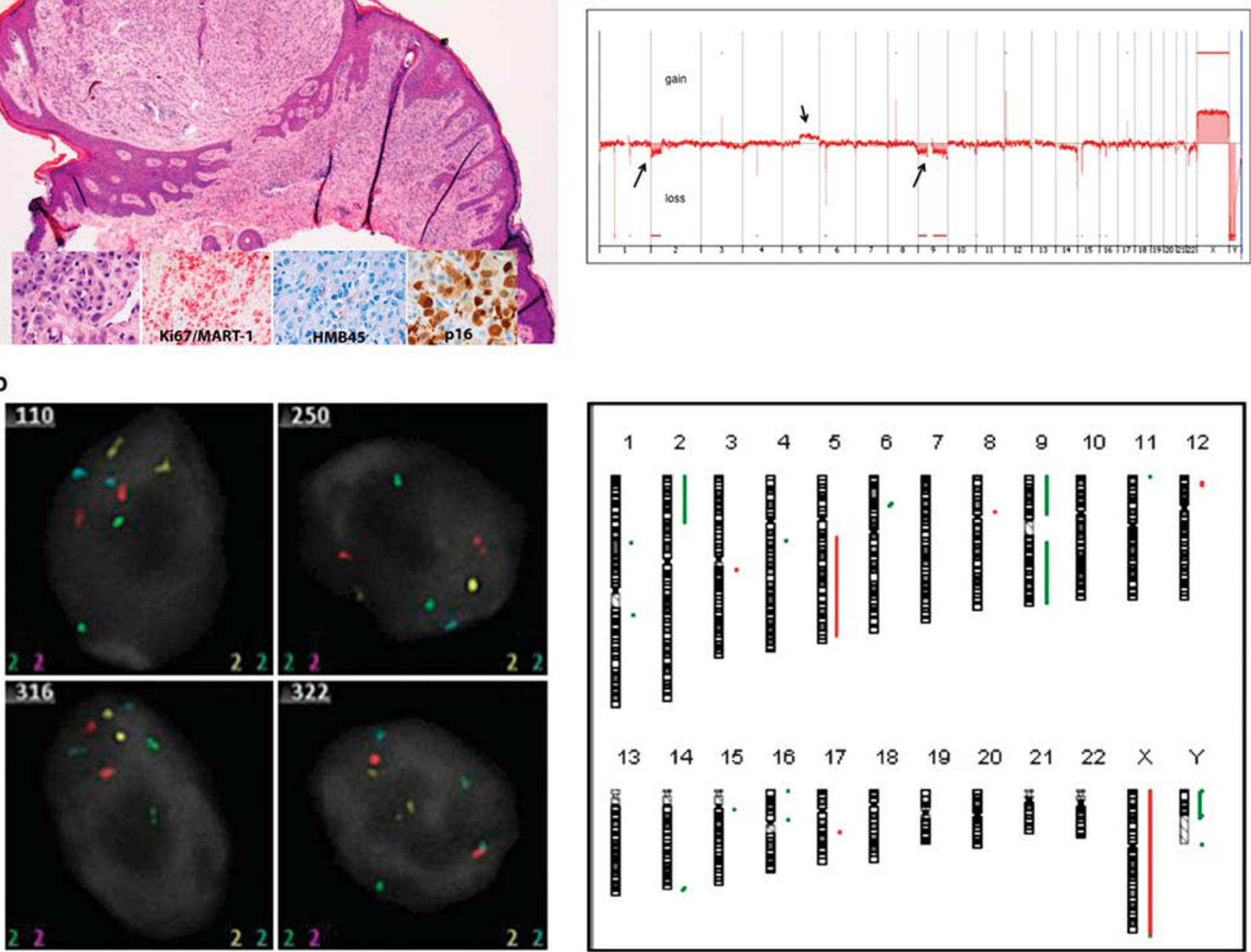

Figure 6 A case of 'spitzoid melanoma' diagnosed by this algorithmic approach. ${ }^{22}$ A 21-year-old woman's cheek lesion showed morphologically atypical spitzoid tumor (a) with deep dermal cell proliferation by a dual-color Ki67/MART-1, a complete loss of HMB45, and an intratumoral heterogeneous loss of p16 expression (Inset). A 4-probe melanoma fluorescence in situ hybridization showed a normal pattern (b). The array-based comparative genomic hybridization assay revealed a loss of chromosome 9 and $2 p$ and a gain of $5 q$ (c). This combinatorial aberration has been frequently found in melanomas and a diagnosis of 'spitzoid melanoma' was rendered.

(low risk of metastasis), or Class 2 (high risk of metastasis), which in combination with sentinel lymph node biopsy, improves the prediction of metastasis risk even in melanomas at an early stage. ${ }^{51}$ These two molecular tests, together with FISH and array-based comparative genomic hybridization, could be potential additional molecular tests in this algorithm for some of eligible atypical spitzoid tumors in the near future.

\section{Case presentation}

Two cases are presented here that demonstrate the diagnostic power of this algorithmic evaluation. For the first case, before seeing the molecular cytogenetic results, 6 of 9 expert dermatopathologists favored a spitzoid melanoma diagnosis, one favored a Spitz nevus, and two wanted to see the molecular results for their final diagnosis. For the second case, two experts favored a spitzoid melanoma, based purely on histological grounds.

The first case is a 21 years old female who presented with a pyogenic granuloma-like lesion on her cheek showing a morphologically compound spitzoid tumor with asymmetry, lack of maturation, high-grade cellular atypia, and frequent dermal and deep dermal mitosis (Figure 6a and inset). ${ }^{22}$ Immunohistochemical assays also detected deep dermal cell proliferation (by using a dual-color Ki67/MART-1 assay), a complete loss of HMB45 expression, and an intratumoral heterogeneous loss of p16 expression (Figure 6a, insets). The algorithmic approach utilizing 4-probe melanoma FISH (NeoSITE Melanoma in 2012) was negative for chromosomal aberrations (Figure 6b). Hence, the array-based comparative 


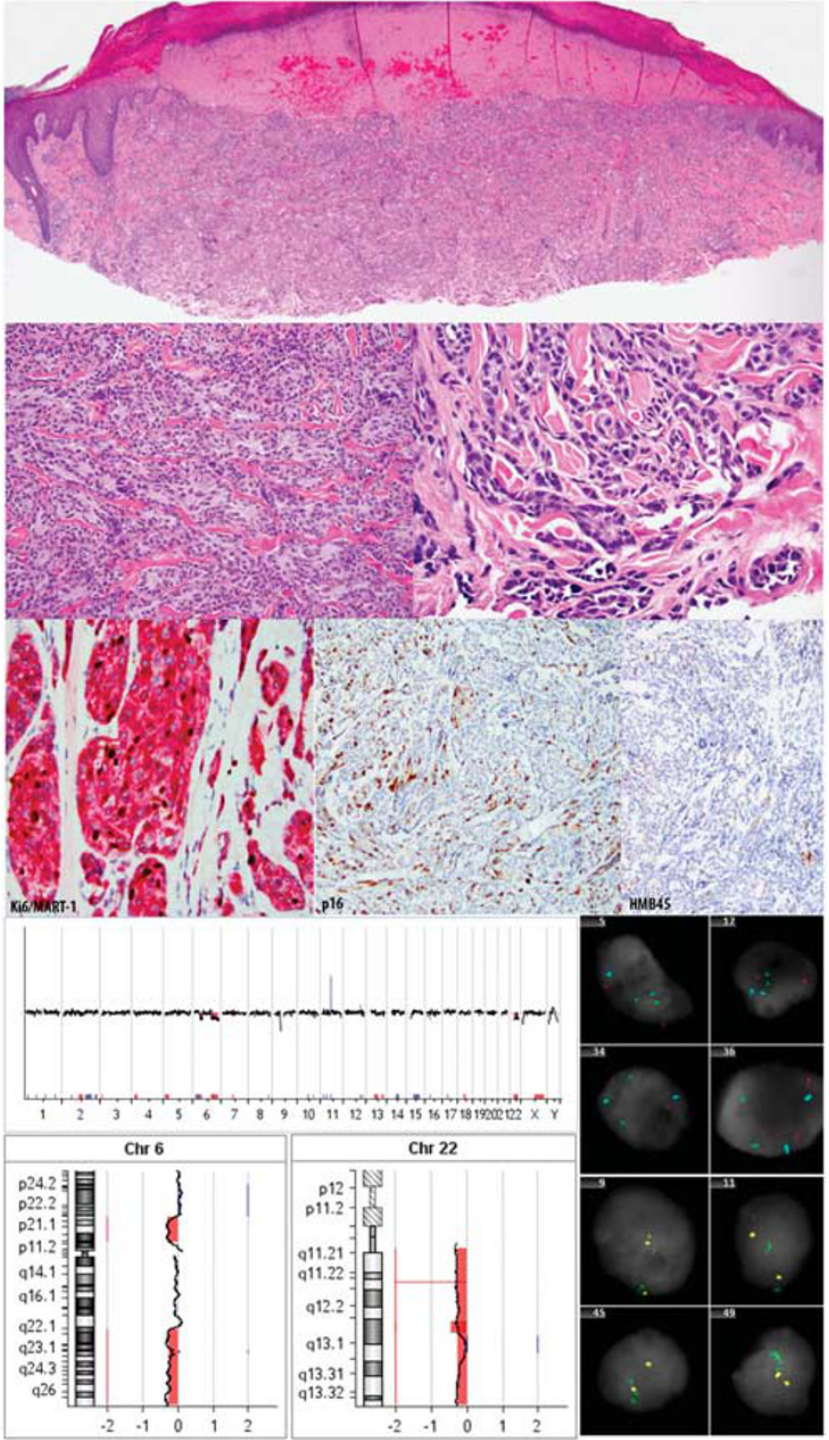

Figure 7 A case of 'atypical spitzoid tumor' in a 14-year-old boy's shoulder, which showed an ulcer, asymmetry, lack of maturation, high-grade cellular atypia, and frequent dermal and deep dermal mitosis. The immunohistochemistry also revealed deep dermal cell proliferation by a dual-color Ki67/MART-1, a complete loss of HMB45, and a near complete loss of p16 expression. A 5-probe melanoma fluorescence in situ hybridization (right bottom) was negative for chromosomal aberrations, but array-based comparative genomic hybridization revealed losses of pericentromeric chromosome 6p, chromosome 6q, and 22q (left bottom). As this combination of chromosomal losses was not known to be typical of melanoma, the tumor was diagnosed as an 'atypical spitzoid tumor.'

genomic hybridization assay was followed and it was observed that the tumor carried a loss of chromosomes 9 and $2 p$ and a gain of $5 q$ (Figure 6c). This combinatory aberration has been frequently found in melanomas and the case was diagnosed as 'spitzoid melanoma'. Following a melanoma protocol, the patient underwent a wide excision and sentinel lymph node dissection, all of which were negative for melanoma. There was no evidence of melanoma after 4 years follow-up.

The second case is a 14 years old boy who presented with a rapidly growing a red nodule $(>1 \mathrm{~cm})$ on the shoulder, which was an ulcerated compound spitzoid tumor with apparent asymmetry, lack of maturation, high-grade cellular atypia, and frequent dermal and deep dermal mitosis (Figure 7). Algorithmic immunohistochemical studies showed deep dermal cell proliferation (by a dualcolor Ki67/MART-1 assay), a complete loss of HMB45, and a near complete loss of p16 expression (Figure 7). The 5-probe melanoma FISH was performed and was negative for the tested chromosomal aberrations. Array-based comparative genomic hybridization, however, revealed that the tumor carried a pericentromeric loss of chromosome $6 \mathrm{p}$, chromosome 6q, and 22q (Figure 7). As this combination of chromosomal losses is not typical of melanomas, the tumor was diagnosed as an 'atypical spitzoid tumor with losses of pericentromeric chromosome 6p, chromosome 6q, and 22q'. For this particular case, a possible association with 'a low clinical risk' was also suggested, probably similar to negative FISH atypical spitzoid tumors, as both FISH and array-based comparative genomic hybridization assays did not display known melanoma molecular signatures. A conservative reexcision of the prior biopsy site and a long-term follow-up were recommended due to the novel nonmelanoma pattern of chromosomal losses.

\section{Conclusions}

The diagnostic algorithm for atypical spitzoid tumors proposed here was designed for routine clinical dermatopathology practice and contributes to a better delineation of atypical spitzoid tumor from spitzoid melanoma and to the prediction of the clinical behavior of atypical spitzoid tumors. Recent advances in cytogenetic/molecular information on atypical spitzoid tumors will help classify a specific subtype of atypical spitzoid tumors with distinct clinical and morphological features and prognosis. It would be important to collect well-annotated atypical spitzoid tumor cases with cytogenetic signatures to create a nationwide or worldwide atypical spitzoid tumor registry, with a further aim to elucidate the molecular classification of atypical spitzoid tumors.

In my opinion, this diagnostic algorithm proposed is still evolving and can be improved from the incorporation of novel molecular genetic advances in atypical spitzoid tumors. The potential applications of this diagnostic algorithm are being also tested in our practice for the evaluation of a variety of ambiguous/borderline nonspitzoid melanocytic tumors in adults. I cannot overemphasize the fact that correlation between histological, immunohistochemical, and cytogenetic/molecular results, and 
available clinical information is an integral part of this diagnostic algorithm for atypical spitzoid tumors.

\section{Acknowledgments}

The reports of the two cases presented here and illustrated in Figures 6 and 7 have been published in Am J Dermatopathol 2013, 35:277-280 and have been presented as a poster in American Society of Dermatopathology 52nd annual meeting, respectively. Some of this review content has been presented as a poster in United States and Canadian Academy of Pathology 2016 annual meeting. I would like to thank Dr. Lauren Clark, the director of Myriad Genetics and Dr. Robert Gasparini, the former chief scientific officer of Neogenomics Laboratories for their kind and detailed information on 'Myriad myPath Melanoma' and 'melanoma FISH' assays, respectively.

\section{Disclosure/conflict of interest}

The author declares no conflict of interest.

\section{References}

1 Spitz S. Melanomas of childhood. Am J Pathol 1948;24: 591-609.

2 Barnhill RL, Argenyi ZB, From L et al. Atypical Spitz nevi/tumors: lack of consensus for diagnosis, discrimination from melanoma, and prediction of outcome. Hum Pathol 1999;30:513-520.

3 Gerami P, Busam K, Cochran A et al. Histomorphologic assessment and interobserver diagnostic reproducibility of atypical spitzoid melanocytic neoplasms with long-term follow-up. Am J Surg Pathol 2014;38: 934-940.

4 Bastian BC, Wesselmann U, Pinkel D, Leboit PE. Molecular cytogenetic analysis of Spitz nevi shows clear differences to melanoma. J Invest Dermatol 1999;113:1065-1069.

5 Bastian BC, Olshen AB, LeBoit PE, Pinkel D. Classifying melanocytic tumors based on DNA copy number changes. Am J Pathol 2003;163:1765-1770.

6 Bastian BC, LeBoit PE, Pinkel D. Mutations and copy number increase of HRAS in Spitz nevi with distinctive histopathological features. Am J Pathol 2000;157: 967-972.

7 Crotty KA, Scolyer RA, Li L et al. Spitz naevus versus Spitzoid melanoma: when and how can they be distinguished? Pathology 2002;34:6-12.

8 Bergman R, Malkin L, Sabo E, Kerner H. MIB-1 monoclonal antibody to determine proliferative activity of Ki-67 antigen as an adjunct to the histopathologic differential diagnosis of Spitz nevi. J Am Acad Dermatol 2001;44:500-504.

9 George E, Polissar NL, Wick M. Immunohistochemical evaluation of p16INK4A, E-cadherin, and cyclin D1 expression in melanoma and Spitz tumors. Am J Clin Pathol 2010;133:370-379.
10 Stefanaki C, Stefanaki K, Antoniou C et al. Cell cycle and apoptosis regulators in Spitz nevi: comparison with melanomas and common nevi. J Am Acad Dermatol 2007;56:815-824.

11 Paradela S, Fonseca E, Pita S et al. Spitzoid melanoma in children: clinicopathological study and application of immunohistochemistry as an adjunct diagnostic tool. J Cutan Pathol 2009;36:740-752.

12 Al Dhaybi R, Agoumi M, Gagne I et al. p16 expression: a marker of differentiation between childhood malignant melanomas and Spitz nevi. J Am Acad Dermatol 2011;65:357-363.

13 Garrido-Ruiz MC, Requena L, Ortiz $\mathrm{P}$ et al. The immunohistochemical profile of Spitz nevi and conventional (non-Spitzoid) melanomas: a baseline study. Mod Pathol 2010;23:1215-1224.

14 Puri PK, Ferringer TC, Tyler WB et al. Statistical analysis of the concordance of immunohistochemical stains with the final diagnosis in spitzoid neoplasms. Am J Dermatopathol 2011;33:72-77.

15 Curtin JA, Fridlyand J, Kageshita T et al. Distinct sets of genetic alterations in melanoma. $N$ Engl J Med 2005;353:2135-2147.

16 Liggett WH Jr., Sidransky D. Role of the p16 tumor suppressor gene in cancer. J Clin Oncol 1998;16: 1197-1206.

17 Witkiewicz AK, Knudsen KE, Dicker AP, Knudsen ES. The meaning of p16(ink4a) expression in tumors: functional significance, clinical associations and future developments. Cell cycle 2011;10:2497-2503.

18 Piepkorn M. Melanoma genetics: an update with focus on the CDKN2A(p16)/ARF tumor suppressors. J Am Acad Dermatol 2000;42:705-722 quiz 23-6.

19 Ward KA, Lazovich D, Hordinsky MK. Germline melanoma susceptibility and prognostic genes: a review of the literature. J Am Acad Dermatol 2012;67: 1055-1067.

20 Romagosa C, Simonetti S, Lopez-Vicente L et al. p16 (Ink4a) overexpression in cancer: a tumor suppressor gene associated with senescence and highgrade tumors. Oncogene 2011;30:2087-2097.

21 Kefford R, Bishop JN, Tucker M et al. Genetic testing for melanoma. Lancet Oncol 2002;3:653-654.

22 DiSano K, Tschen JA, Cho-Vega JH. Intratumoral heterogeneity of chromosome 9 loss and CDKN2A (p16) protein expression in a morphologically challenging spitzoid melanoma. Am J Dermatopathol 2013;35: 277-280.

23 Bogdan I, Burg G, Boni R. Spitz nevi display allelic deletions. Arch Dermatol 2001;137:1417-1420.

24 Sini MC, Manca A, Cossu A et al. Molecular alterations at chromosome 9p21 in melanocytic naevi and melanoma. Br J Dermatol 2008;158:243-250.

25 Yazdan P, Cooper C, Sholl LM et al. Comparative analysis of atypical spitz tumors with heterozygous versus homozygous 9p21 deletions for clinical outcomes, histomorphology, BRAF mutation, and p16 expression. Am J Surg Pathol 2014;38: 638-645.

26 Scholzen T, Gerdes J. The Ki-67 protein: from the known and the unknown. J Cell Physiol 2000;182: 311-322.

27 Perez-Cadahia B, Drobic B, Davie JR. H3 phosphorylation: dual role in mitosis and interphase. Biochem Cell Biol 2009;87:695-709.

28 Schimming TT, Grabellus F, Roner $\mathrm{M}$ et al. pHH3 immunostaining improves interobserver agreement of 
mitotic index in thin melanomas. Am J Dermatopathol 2012;34:266-269.

29 Gown AM, Vogel AM, Hoak D et al. Monoclonal antibodies specific for melanocytic tumors distinguish subpopulations of melanocytes. Am J Pathol 1986;123: 195-203.

30 Kamino H. Spitzoid melanoma. Clin Dermatol 2009;27: $545-555$

31 Gerami P, Jewell SS, Morrison LE et al. Fluorescence in situ hybridization (FISH) as an ancillary diagnostic tool in the diagnosis of melanoma. Am J Surg Pathol 2009;33:1146-1156.

32 Gerami P, Li G, Pouryazdanparast P et al. A highly specific and discriminatory FISH assay for distinguishing between benign and malignant melanocytic neoplasms. Am J Surg Pathol 2012;36:808-817.

33 North JP, Garrido MC, Kolaitis NA et al. Fluorescence in situ hybridization as an ancillary tool in the diagnosis of ambiguous melanocytic neoplasms: a review of 804 cases. Am J Surg Pathol 2014;38: 824-831.

34 Gammon B, Beilfuss B, Guitart J, Gerami P. Enhanced detection of spitzoid melanomas using fluorescence in situ hybridization with 9p21 as an adjunctive probe. Am J Surg Pathol 2012;36:81-88.

35 Gaiser T, Kutzner H, Palmedo G et al. Classifying ambiguous melanocytic lesions with FISH and correlation with clinical long-term follow up. Mod Pathol 2010;23:413-419.

36 Vergier B, Prochazkova-Carlotti M, de la Fouchardiere A et al. Fluorescence in situ hybridization, a diagnostic aid in ambiguous melanocytic tumors: European study of 113 cases. Mod Pathol 2011;24:613-623.

37 Kerl K, Palmedo G, Wiesner T et al. A proposal for improving multicolor FISH sensitivity in the diagnosis of malignant melanoma using new combined criteria. Am J Dermatopathol 2012;34:580-585.

38 Gerami P, Mafee M, Lurtsbarapa T et al. Sensitivity of fluorescence in situ hybridization for melanoma diagnosis using RREB1, MYB, Cep6, and 11q13 probes in melanoma subtypes. Arch Dermatol 2010;146:273-278.

39 Massi D, Cesinaro AM, Tomasini C et al. Atypical Spitzoid melanocytic tumors: a morphological, mutational, and FISH analysis. J Am Acad Dermatol 2011;64:919-935.
40 Gerami P, Scolyer RA, Xu X et al. Risk assessment for atypical spitzoid melanocytic neoplasms using FISH to identify chromosomal copy number aberrations. Am J Surg Pathol 2013;37:676-684.

41 Shen L, Cooper C, Bajaj S et al. Atypical spitz tumors with 6q23 deletions: a clinical, histological, and molecular study. Am J Dermatopathol 2013;35:804-812.

42 Barrett MT, Scheffer A, Ben-Dor A et al. Comparative genomic hybridization using oligonucleotide microarrays and total genomic DNA. Proc Natl Acad Sci USA 2004;101:17765-17770.

43 Bastian BC, LeBoit PE, Hamm H et al. Chromosomal gains and losses in primary cutaneous melanomas detected by comparative genomic hybridization. Cancer Res 1998;58:2170-2175.

44 Harvell JD, Kohler S, Zhu S et al. High-resolution arraybased comparative genomic hybridization for distinguishing paraffin-embedded Spitz nevi and melanomas. Diagn Mol Pathol 2004;13:22-25.

45 Ali L, Helm T, Cheney $\mathrm{R}$ et al. Correlating array comparative genomic hybridization findings with histology and outcome in spitzoid melanocytic neoplasms. Int J Clin Exp Pathol 2010;3:593-599.

46 Wang L, Rao M, Fang Y et al. A genome-wide highresolution array-CGH analysis of cutaneous melanoma and comparison of array-CGH to FISH in diagnostic evaluation. J Mol Diagn 2013;15:581-591.

47 Raskin L, Ludgate M, Iyer RK et al. Copy number variations and clinical outcome in atypical spitz tumors. Am J Surg Pathol 2011;35:243-252.

48 Wiesner T, He J, Yelensky $\mathrm{R}$ et al. Kinase fusions are frequent in Spitz tumours and spitzoid melanomas. Nat Commun 2014;5:3116.

49 Wiesner T, Murali R, Fried I et al. A distinct subset of atypical Spitz tumors is characterized by BRAF mutation and loss of BAP1 expression. Am J Surg Pathol 2012;36:818-830.

50 Clarke LE, Warf BM, Flake DD 2nd et al. Clinical validation of a gene expression signature that differentiates benign nevi from malignant melanoma. J Cutan Pathol 2015;42:244-252.

51 Gerami P, Cook RW, Russell MC et al. Gene expression profiling for molecular staging of cutaneous melanoma in patients undergoing sentinel lymph node biopsy. J Am Acad Dermatol 2015;72:780-785.e3. 